\title{
1 Multiple indirect ELISAs for serological detection of SARS-CoV-2 antibodies
}

3 Abdullah Algaissi ${ }^{1,2,3, \#}$, Mohamed A Alfaleh ${ }^{1,4, \#}$, Sharif Hala ${ }^{5,6, \#}$, Turki S Abujamel ${ }^{1,7}$, Sawsan S 4 Alamari $^{1,8}$, Khalid A Alluhaybi ${ }^{1,4}$, Haya I Hobani ${ }^{1}$, Reem M Alsulaiman ${ }^{1}$, Rahaf H AlHarbi ${ }^{1,9}$, 5 Mohammad Z El-Assouli ${ }^{1}$, Wesam H Abdulaal ${ }^{8}$, Afrah A AL-Somali ${ }^{10}$, Fadwa S Alofi ${ }^{11}$, Asim A 6 Khogeer $^{12}$, Almohanad A Alkayyal ${ }^{13}$, Ahmad Bakur Mahmoud ${ }^{14}$, Naif AM Almontashiri ${ }^{15}$, Arnab $7 \quad$ Pain $^{5,16,17}$, Anwar M Hashem ${ }^{1,18, *}$

$9{ }^{1}$ Vaccines and Immunotherapy Unit, King Fahd Medical Research Center; King Abdulaziz 10 University, Jeddah, Saudi Arabia

112 Department of Medical Laboratories Technology, College of Applied Medical Sciences, Jazan 12 University, Jazan, Saudi Arabia

$13{ }^{3}$ Medical Research Center, Jazan University, Jazan, Saudi Arabia

$14{ }^{4}$ Faculty of Pharmacy, King Abdulaziz University, Jeddah, Saudi Arabia

155 Pathogen Genomics Laboratory, Division of Biological and Environmental Sciences and 16 Engineering (BESE), Thuwal, Makkah, Saudi Arabia

$17{ }^{6}$ King Abdullah International Medical Research Centre, King Saud University for Health

18 Sciences, Ministry of National Guard Health Affairs, Jeddah, Saudi Arabia

$19{ }^{7}$ Department of Medical Laboratory Technology, Faculty of Applied Medical Sciences, King 20 Abdulaziz University, Jeddah, Saudi Arabia

$21{ }^{8}$ Department of Biochemistry, Faculty of Science, King Abdulaziz University, Jeddah, Saudi

22 Arabia

$23{ }^{9}$ Department of Biology, faculty of science, King Abdulaziz university, jeddah, saudi arabia 
$24{ }^{10}$ Infectious Diseases Department, King Abdullah medical complex, Jeddah, Saudi Arabia

$25{ }^{11}$ Infectious Diseases Department, King Fahad Hospital, Almadinah Almunwarah, Saudi Arabia

$26{ }^{12}$ Plan and Research Department, General Directorate of Health Affairs Makkah Region, Ministry

27 of Health, Makkah, Saudi Arabia

$28{ }^{13}$ Department of Medical Laboratory Technology, University of Tabuk, Tabuk, Saudi Arabia

2914 College of Applied Medical Sciences, Taibah University, Almadinah Almunwarah, Saudi 30 Arabia

$31{ }^{15}$ Center for Genetics and Inherited Diseases, Taibah University, Almadinah Almunwarah, Saudi 32 Arabia

$33{ }^{16}$ Global Institution for Collaborative Research and Education (GI-CoRE), Hokkaido University,

34 N20 W10 Kita-ku, Sapporo, Japan

$35{ }^{17}$ Nuffield Division of Clinical Laboratory Sciences (NDCLS), University of Oxford, Oxford, 36 OX3 9DU, UK

$37{ }^{18}$ Department of Medical Microbiology and Parasitology, Faculty of Medicine, King Abdulaziz 38 University, Jeddah, Saudi Arabia

39 \# equal contribution

40

41 * Corresponding author: Anwar M Hashem; Faculty of Medicine and King Fahd Medical 42 Research Center, King Abdulaziz University, P.O.Box 80205, Jeddah 21859, Saudi Arabia. Tel. 43 +966 (12) 6400000 ext. 21033, E-mail: amhashem@kau.edu.sa.

45 Running title: Validated ELISAs for COVID-19 antibody response detection 


\section{Abstract}

47 As the coronavirus disease 2019 (COVID-19), which is caused by the novel coronavirus SARS-

$48 \mathrm{CoV}-2$, continues to spread rapidly around the world, there is an urgent need for validated

49 serological assays to evaluate viral specific antibody responses in COVID-19 patients or recovered

50 individuals. In this study, we established and used indirect Enzyme Linked Immunosorbent Assay

51 (ELISA)-based serological tests to study the antibody response in COVID-19 patients. In order to

52 validate the assays, we determined the cut-off values, sensitivity and specificity of the developed

53 assays using sera collected from COVID-19 patients in Saudi Arabia at different time points after

54 disease onset, as well as sera that are seropositive to other human CoVs; namely MERS-CoV,

55 hCoV-OC43, hCoV-NL63, hCoV-229E, and hCoV-HKU1. The SARS-CoV-2 S1 subunit of the

56 spike glycoprotein and nucleocapsid (N) ELISAs that we developed here not only showed high

57 specificity and sensitivity, but also did not show any cross-reactivity with other CoVs. We also

58 showed that all RT-PCR confirmed COVID-19 patients included in our study developed both virus

59 specific IgM and IgG as early as one week after the onset of disease. The availability of these

60 validated assays will enable us to determine the nature and duration of the antibody response

61 mounted in response to SARS-CoV-2 infection. It will also allow conducting large-scale

62 epidemiological studies to determine evidence of previous exposure to the virus and assess the true

63 extent of virus spread within communities. 


\section{Introduction}

65 In December 2019, a cluster of atypical pneumonia was reported in Wuhan City, the capital of

66 Hubei province in China. The etiological agent was quickly identified as a novel coronavirus,

67 subsequently named as severe acute respiratory syndrome coronavirus 2 (SARS-CoV-2), and

68 identified as a cause of the Coronavirus Disease 2019 (COVID-19) [1]. Within weeks of its

69 discovery, SARS-CoV-2 has rapidly spread to more than 200 countries around the world, causing

70 large scale morbidity and mortality. Eventually, it was recognized as a pandemic by the World

71 Health Organization (WHO) in early March of 2020. The rapid and continued spread of the virus

72 has triggered the implementation of unprecedented public health measures by affected countries,

73 including travel bans, borders closure, enforced curfew, lockdown of cities and shutdown of most

74 businesses, public gatherings and other activities. Nevertheless, the spread of the virus was further

75 complicated by the absence of vaccines and specific therapeutics to date.

77 Coronaviruses (CoVs) are a large group of viruses that can infect a wide range of hosts, including

78 humans, animals and birds [2]. They are classified into four genera; alpha, beta, gamma and delta,

79 in which only viruses from alphacoronaviruses (alpha-CoVs) and betacoronaviruses (beta-CoV)

80 were recognized to infect humans so far [3]. SARS-CoV-2 belongs to the beta-CoV genus, which

81 also contains two other highly pathogenic human CoVs; SARS-CoV and MERS-CoV as well as a

82 number of animal CoVs [4]. Genome sequence analysis shows that SARS-CoV-2 shares nearly

$8379.5 \%$ identity with SARS-CoV and $\sim 96 \%$ with a bat SARS-like CoV [1]. CoVs are enveloped

84 viruses with a positive-sense, single-stranded, $\sim 30 \mathrm{~kb}$ RNA genome, which contains at least 6 open

85 reading frames (ORFs) [4]. The first two-thirds of the genome encodes for polyproteins: ppla and

$86 \mathrm{pplab}$ that are processed by viral and host proteases into 16 non-structural proteins (nsp1-16) [4,5]. 
87 The other third of the genome encodes the four main structural proteins (envelope (E), membrane

88 (M), spike (S), and nucleocapsid (N) proteins) as well as other accessory proteins [4,5].

90 As SARS-CoV-2 continues to spread around the globe, it is important to understand the duration

91 and nature of immunity mounted in response to infection, which is currently not fully understood

92 and investigated. Furthermore, the actual extent of the current global COVID-19 pandemic is not

93 well known; therefore, serological assays are critically needed to shed light on all these

94 unanswered questions. Here, we report the development and validation of multiple indirect

95 ELISA-based serological assays that can be adapted and used by laboratories to determine the

96 immune status of individuals in surveillance and epidemiological studies, as we have previously

97 described for MERS-CoV [6,7]. Using sera derived from either confirmed COVID-19 patients or

98 known noninfected healthy controls, we validated our ELISAs and determined their cut off values,

99 sensitivity and specificity. We also showed that our assays had no cross-reactivity using sera with

100 known positivity to MERS-CoV and other common CoVs. Our study shows that SARS-CoV-2

101 IgM or IgG specific antibodies for either SARS-CoV-2 S1 or N antigens can be detected virtually

102 in all real-time polymerase chain reaction (RT-PCR) confirmed COVID-19 patients included in

103 our study as early as one week after disease-onset. Antibodies levels sharply increased by week

104 two, with IgG persisting through week four compared to IgM, which peaked by week 2 or 3 before 105 declining as previously shown [8]. 
Material and methods

107

108 Samples

109 A 100 serum samples from healthy controls collected before the COVID-19 pandemic with a 110 positive control from a confirmed COVID-19 patient were used to determine the cut-off values for 111 the developed indirect ELISAs. Another set of samples including 8 SARS-CoV-2 and MERS-CoV 112 seronegative samples, two MERS-CoV seropositive samples and three SARS-CoV-2 seropositive 113 samples were used to determine the cross-reactivity of the assays. A third cohort of pre-pandemic 114 samples $(\mathrm{n}=125)$ and RT-PCR confirmed COVID-19 patients $(\mathrm{n}=52)$ including samples 115 collected during the $1^{\text {st }}$ week $(n=10), 2^{\text {nd }}$ week $(n=23), 3^{\text {rd }}$ week $(n=14)$ or $4^{\text {th }}$ week $(n=5)$ of 116 symptoms-onset were used to evaluate the developed ELISAs. All samples were obtained from 117 multi ethnicity patients or donors residing in Saudi Arabia. All samples were anonymized and used 118 based on ethical approvals obtained from the Unit of Biomedical Ethics in King Abdulaziz 119 University Hospital (Reference No 245-20), the Institutional Review Board at the Ministry of 120 Health, Saudi Arabia (IRB Numbers: H-02-K-076-0320-279 and H-02-K-076-0420-285), and the

121 Global Center for Mass Gatherings Medicine (GCMGM) (No. 20/03A).

123 Expression and production of SARS-CoV-2 recombinant proteins.

124 Recombinant SARS-CoV-2 S1 submit of the S protein (amino acids 1-685), MERS-CoV S1 125 subunit (amino acids 1-725), and full-length S proteins from hCoV-OC43, hCoV-NL63, hCoV126 229E and hCoV-HKU1 viruses tagged with histidine tag were purchased commercially (Sino 127 Biological, China). Recombinant SARS-CoV-2 and MERS-CoV N proteins were expressed and 128 purified from Escherichia coli BL21 (DE3) cells using a nickel-nitrilotriacetic acid (Ni-NTA) 
129 column according to the manufacturer's protocol and as previously described [6]. Positive fractions

130 of $\mathrm{N}$ proteins were pooled, aliquoted and stored at $-80^{\circ} \mathrm{C}$ until used. SARS-CoV-2 proteins were

131 confirmed by Western blot using anti-His tag antibodies as well as SARS-CoV-2 seropositive and

132 seronegative human serum samples as previously described [6].

133

\section{Indirect ELISA}

135 Recombinant SARS-CoV-2 S1, MERS-CoV S1 and full-length S proteins from other human CoVs

136 at a concentration of $1 \mu \mathrm{g} / \mathrm{mL}$ in phosphate-buffered saline (PBS) were used to coat 96-well high

137 binding ELISA plates (Greiner Bio One, Monroe, NC) with $50 \mu \mathrm{L}$ per well. Similarly, in-house

138 produced SARS-CoV-2 and MERS-CoV N proteins were used to coat plates at a concentration of

$1394 \mu \mathrm{g} / \mathrm{mL}$. All plates were coated for overnight at $4^{\circ} \mathrm{C}$, washed three times with PBS containing

$140 \quad 0.05 \%$ tween-20 (PBS-T), and blocked with 5\% skim milk in PBS-T buffer at $37^{\circ} \mathrm{C}$ for $1 \mathrm{~h}$. After

141 blocking, plates were washed three times and incubated with serum samples diluted at 1:100 in

142 PBS-T with 5\% milk for $1 \mathrm{~h}$ at $37^{\circ} \mathrm{C}$. Plates were then washed three times again with PBS-T,

143 incubated with HRP-conjugated goat anti-human $\operatorname{IgG}(\mathrm{H}+\mathrm{L})$ or $\operatorname{IgM}$ antibodies (Jackson

144 ImmunoResearch, West Grove, PA) for $1 \mathrm{~h}$, washed again, and incubated with TMB (3,3',5,5' -

145 tetramethylbenzidine) substrate (KPL, Gaithersburg, MD) at $37^{\circ} \mathrm{C}$ for $30 \mathrm{~min}$. The reaction was

146 terminated by adding $100 \mu \mathrm{L}$ per well of the ELISA stop solution ( $0.16 \mathrm{M}$ sulfuric acid). The

147 absorbance was measured at $450 \mathrm{~nm}$ using the ELx808 ${ }^{\mathrm{TM}}$ Absorbance Microplate Reader

148 (BioTek, Winooski, VT).

149

150

151 


\section{Statistical analysis}

153 The sensitivity of each ELISA was determined as (the number of samples that are true positives /

154 the total number of samples that are true positives and false negatives $\times 100$ ), and the specificity 155 was determined as (the number of samples that are true negatives / the total number of samples 156 that are true negatives and false positives $) \times 100$. Receiver operating characteristic $($ ROC $)$ analysis 157 was calculated using GraphPad Prism V8 software (GraphPad Co.). Sensitivity, specificity and 158 ROC analysis were calculated based on RT-PCR results. Each experiment was done twice with 159 each serum sample run in duplicates.

160 


\section{Results}

162

163 Expression and production of SARS-CoV-2 proteins

164 The S protein of SARS-CoV-2 is a major immunogen and is divided into two subunits; S1 which 165 contains the receptor binding domain (RBD) and S2 that mediates the fusion with the host 166 membrane [9]. The $\mathrm{N}$ protein is another target for most serological assays for CoVs because of its 167 abundant expression $[5,6,10]$. We and others have shown that both proteins are suitable and 168 comparable for the detection of virus-specific antibodies in MERS patients [6,10]. In this study, 169 we have successfully expressed and purified a His-tagged SARS-CoV-2 N protein and 170 subsequently used it for indirect ELISA development. Recombinant N protein was induced and 171 expressed upon induction with IPTG, and purified on the Ni-NTA affinity chromatography 172 column, while the recombinant S1-His tagged protein was purchased commercially. Western blot 173 analysis showed that both $\mathrm{S} 1(\sim 110 \mathrm{KDa}$, Figure 1a) and N ( 46 KDa, Figure 1b) proteins were 174 detected using anti-His antibodies and shown to bind specifically to sera derived from COVID-19 175 patients but not to COVID-19 seronegative sera from normal human donors collected prior to the 176 pandemic. These data indicate that both $\mathrm{S} 1$ and $\mathrm{N}$ proteins are antigenically similar to native 177 proteins and able to strongly and specifically detect SARS-CoV-2 antibodies in serum samples. Development, optimization and determination of the cut-off values of the indirect ELISAs

180 We developed four different types of indirect ELISAs for the testing of IgM and IgG antibodies 181 using purified SARS-CoV-2 S1 and N proteins as coating antigens. We initially optimized the 182 coating conditions for the ELISA using known SARS-CoV-2 seronegative and seropositive sera 183 and found that the optimal working concentrations of each antigen were $1 \mu \mathrm{g} / \mathrm{mL}$ and $4 \mu \mathrm{g} / \mathrm{mL}$ for 
184 recombinant $\mathrm{S} 1$ and $\mathrm{N}$ proteins, respectively (data not shown). Furthermore, optimal serum

185 dilution was determined using checkerboard titration where the highest OD ratio values of positive 186 to negative samples $(\mathrm{P} / \mathrm{N})$ were obtained. After optimization, we tested sera from 100 normal 187 human donors and one serum sample from an RT-PCR confirmed COVID-19 patient in the 188 developed ELISAs at a dilution of 1:100 to determine the cut-off values (mean $+3 \mathrm{SD})$. As shown 189 in Figure 2, the cut-off values were found to be 0.17 (mean $=0.09, \mathrm{SD}=0.3$ ) for S1 IgG-ELISA, $1900.30($ mean $=0.09, \mathrm{SD}=0.07)$ for S1 IgM-ELISA, $0.40($ mean $=0.17, \mathrm{SD}=0.08)$ for $\mathrm{N}$ IgG191 ELISA, and $0.55($ mean $=0.24, \mathrm{SD}=0.10)$ for $\mathrm{N}$ IgM-ELISA. Almost all tested samples were 192 below the determined cut-off values suggesting high specificity of the assays.

\section{Determination of potential cross-reactivity with other CoVs}

195 The ability of our developed assay to specifically detect and significantly differentiate SARS-CoV1962 antibodies in patients that might be co-infected with other CoVs was assessed. Here, ELISA 197 plates were coated with different antigens representing MERS-CoV1 (S1 and N proteins) and the 198 S protein of the other human CoVs, including hCoV-OC43, hCoV-NL63, hCoV-229E and hCoV199 HKU1 at a concentration of $1 \mu \mathrm{g} / \mathrm{mL}$. Using sera with known seropositivity to either MERS-CoV 200 or to other known human CoVs, we found that our developed SARS-CoV-2 S1 and N-based 201 ELISAs for IgG and IgM can only detect antibodies from COVID-19 seropositive sera but not 202 from any of the other tested serum samples that are known to be IgG seropositive for MERS-CoV, $203 \mathrm{hCoV}-\mathrm{OC} 43, \mathrm{hCoV}-\mathrm{NL} 63, \mathrm{hCoV}-229 \mathrm{E}$ or hCoV-HKU1 (Figure 3). On the other hand, using S1 204 and $\mathrm{N}$ antigens of MERS-CoV only detected antibodies from MERS seropositive samples but not 205 others. As expected, using S protein from other human CoVs showed presence of IgG antibodies 206 only in all tested serum samples suggesting previous exposure to these common cold viruses. 
207 Collectively, these data show that our assays can specifically detect and significantly differentiate

208 SARS-CoV-2 specific IgG and IgM antibodies from those against other human CoVs in serum

209 samples.

210

211 Validation of the developed ELISAs and testing of seroconversion

212 Testing of serum samples collected from another cohort of healthy donors $(n=125)$ or COVID-

21319 patients $(\mathrm{n}=52)$ showed that our developed ELISAs could detect both IgG and IgM against

214 both antigens as early as week one post symptoms-onset (Figure 4). Our data also show that IgG

215 levels against both antigens increased over time, while IgM levels peaked by week 2 or 3 before

216 starting to decline. Based on these data and on the assumption that all RT-PCR positive patients

217 developed humoral response, we tried to determine the specificity and sensitivity of the developed

218 ELISAs. As shown in Table 1, the specificity of the assays ranged between 91.2\%-97.6\%. The

219 sensitivity, however, was dependent on the sampling time in relevance to disease onset. During

220 the first week post symptoms onset, the sensitivity of IgM and IgG ELISAs ranged between 20\%-

$22130 \%$ and $40 \%-60 \%$, respectively (Table 1). Nonetheless, the sensitivity of the assays increased to

$22288.5 \%, 84.6 \%, 100 \%$ and $88.5 \%$ for S1 IgG-ELISA, S1 IgM-ELISA, N IgG-ELISA and N IgM-

223 ELISA, respectively by week two. Importantly, while these sensitivity values were maintained at

$224100 \%$ for N IgG-ELISA or increased to $100 \%$ for both S1 IgG-ELISA and S1 IgM-ELISA during

225 week three and four post symptoms onset, N IgM-ELISA's sensitivity declined.

226

227 Next, we conducted ROC analysis to examine the diagnostic power of each developed assay as

228 shown in Figure 5. Our analysis showed high accuracy of S1 IgG-ELISA, S1 IgM-ELISA and N

229 IgG-ELISA with overall area under curve (AUC) of $0.938 \pm 0.027$ (95\% CI: 0.886 - 0.990), 0.953 
$230 \pm 0.021(95 \%$ CI: $0.911-0.995)$ and $0.977 \pm 0.015$ (95\% CI: $0.948-1.000)$, respectively, compared 231 to N IgM-ELISA which showed lower AUC of $0.886 \pm 0.037$ (95\% CI: $0.812-0.959)$ (Table 2).

232 It was also clear that the accuracy of these assays was dependent on the sampling time as it was 233 low when testing samples collected during the first week after symptoms onset compared to those 234 collected during or after the second week of onset. Furthermore, high reproducibility was also 235 observed for all assays with very minimal variation (5\%-10\%) in obtained OD values including 236 inter-assay and intra-assay testing conducted on different days or by different individuals (data not 237 shown). 


\section{Discussion}

239 In the current study, we report the development and validation of an ELISA-based serological 240 assays for the detection of SARS-CoV-2 specific IgG and IgM antibodies in COVID-19 serum 241 specimens. We showed that our ELISAs can specifically detect SARS-CoV-2 specific IgM and

242 IgM antibodies in sera from COVID-19 patients, but not from sera derived from healthy human 243 donors. Our data also show that our SARS-CoV-2 S1 and N ELISAs do not cross-react with sera 244 that are seropositive to other human CoVs; including human CoVs that belong to the beta-CoV 245 genus such as MERS-CoV, hCoV-OC43 and hCoV-HKU1, as well as those from alpha-CoVs such 246 as hCoV-NL63 and the hCoV-229. Furthermore, using the developed ELISAs, we evaluated the

247 production of SARS-CoV-2 specific IgG and IgM antibodies in a cohort of hospitalized COVID-

24819 patients $(\mathrm{n}=52)$, including samples collected during the $1^{\text {st }}$ week $(n=10), 2^{\text {nd }}$ week $(n=23)$,

$2493^{\text {rd }}$ week $(n=14)$ or $4^{\text {th }}$ week $(n=5)$ of symptoms-onset. Our analysis showed that SARS-CoV-2

250 IgM or IgG specific antibodies for either SASR-CoV-2 S1 or N antigens can be virtually detected 251 in all RT-PCR confirmed COVID-19 patients in this study. We showed that both virus specific

252 IgG and IgM can be detected as early as one week after disease-onset but significantly increased 253 by week two and three, with IgG persisting through week four (last time point in our study) 254 compared to IgM which peaked by week 2 or 3 before declining. This increase in IgG over time 255 and the decline in IgM antibodies by week 4 are consistent with some recent reports [11-14].

257 To be able to use the developed assays for large scale serosurveys, we determined the cut-off 258 values, specificity and sensitivity of the different developed ELISAs. Our analysis showed that the 259 cut-off values were found to be 0.17 (mean $=0.09, \mathrm{SD}=0.3)$ for S1 IgG-ELISA and 0.30 (mean $260=0.09, \mathrm{SD}=0.07$ ) for $\mathrm{S} 1 \mathrm{IgM}$-ELISA. While for the $\mathrm{N}$ based ELISAs the cut-off values were 
261 found to be $0.40($ mean $=0.17, \mathrm{SD}=0.08)$ and $0.55($ mean $=0.24, \mathrm{SD}=0.10)$ for $\operatorname{IgG}$ and $\operatorname{IgM}$

262 antibodies, respectively. Almost all seronegative samples were below the determined cut-off

263 values, suggesting high specificity of the assays. Our ROC analysis also demonstrated powerful

264 diagnostic performance of the developed assays.

266 The fact that all RT-PCR confirmed COVID-19 patients included in this study developed virus-

267 specific antibody responses should be reassuring. The majority also showed detection of antibody

268 response as early as week one. Although it has not been proven with SARS-CoV-2 in humans

269 whether the mounted antibody response is protective and long-lasting, such responses are likely to

270 be associated with protection from reinfection. Reinfection in humans has not been reported in

271 SARS-CoV or MERS-CoV, and antibody responses against these two viruses were reported to last

272 for up to three years $[15,16]$. Interestingly, a recent report examined the possibility of SARS-CoV-

2732 reinfection in non-human primates and showed that reinfection was unlikely after the induction

274 of antibody responses [17]. Nevertheless, the possibility of reinfection in humans is a pressing

275 question that warrants further investigations. The assays we presented here would be of a great

276 utility not only to conduct such studies but also to examine the longevity of the mounted antibody

277 responses against SARS-CoV-2 infection, which is critical for the vaccine development efforts.

278 Serological assays like the ones we developed should be able to address these questions in the near

279 future. The early detection of specific antibodies in COVID-19 patients also highlights the

280 diagnostic importance of these assays especially in mild cases which usually present late to

281 hospitals or go undetected.

282 
283 Some seropositive COVID-19 sera were also found positive to other low pathogenic human CoVs,

284 which may indicate that previous infections with other CoVs provide no immunity, at least in our

285 cohort of COVID-19 patients. Interestingly, a recent study attempted to understand why SARS-

286 CoV-2 infected children developed less severe symptoms compared to adults, suggested a possible

287 cross-protection due to previous infections with circulating common cold CoVs, mostly through

288 virus-specific T cell responses [18]. While we cannot confirm this suggestion here since the age

289 range of the COVID-19 patients in our study was between 24 to 75 years and we only examined

290 humoral immune responses, future studies clearly need to investigate this possibility further.

291

292 Another important finding in our study is that using both S1 and N in serology could lead to the

293 detection of as many potential seropositive specimens as possible than using any of them alone.

294 This is of great importance amid the current rapid and continuing spread of SARS-CoV-2 and the

295 need for a quick and efficient method for contacts and cases tracing. It is now evident that 296 asymptomatic infections occur and could play an important role in virus spread [19-21]. Thus, the

297 ability to detect asymptomatic or mild cases is crucial for the epidemiological investigation [8,12].

299 Few serological assays have been reported thus far and most of them use the full S protein, S1

300 subunit or the RBD as capture antigens $[8,11-13,22]$. While these assays show high sensitivity and

301 specificity rates, the use of the S1 or the RBD alone may result in missing cases or give less

302 accurate estimation of the mounted antibody response since high levels of antibodies are generated

303 to areas outside S1 or RBD [23]. Additionally, as it mediates binding and entry into cells, the S

304 protein is under continuous selective pressure, which makes it more prone to acquire mutations

305 that might affect the accuracy of S-based serological assays [24]. In our assays, we included N- 
306 based ELISA in addition to S1 and found them complementary to each other with both showing

307 high sensitivity and specificity. Another reason to include N-based ELISA in the serological

308 testing algorithm is its relatively small size and lack of glycosylation sites, which makes it easy to

309 clone and produce in prokaryotic expression systems, especially in resource-limited settings [3].

310 We believe that using both S1 and N ELISAs would capture as many potential SARS-CoV-2

311 positive cases as possible than using any of them alone.

312

313 The current standard method for the detection of SARS-CoV-2 relies on the detection of the viral

314 RNA during the acute phase of the disease by RT-PCR. Although this highly sensitive method can

315 effectively detect SARS-CoV-2 infection during acute infection phase, RT-PCR is time consuming

316 and has limited detection rate of virus beyond week 3 after symptoms-onset $[25,26]$. Some of these

317 issues could be addressed by the availability of well validated serological assays. Moreover, the

318 development of serological assays is an essential step for the understanding of the epidemiology

319 of SARS-CoV-2 infection. Of note, while our study reports a well validated ELISA assays, we

320 have not assessed virus neutralization activities of detected antibodies. However, recent studies

321 have shown positive correlation between high titers of IgG antibodies detected by ELISAs with

322 neutralizing antibodies [22].

323

324 We believe that our assays are well validated, highly specific, sensitive and can be used for

325 serosurveys to inform us about the extent of the current spread of COVID-19 pandemic in the

326 population. Such studies are also important for a better understanding of the nature of the immune

327 response to SARS-CoV-2, and the true estimate of the attack and infection fatality rates in different

328 human populations. 


\section{$329 \quad$ Funding}

330 We wish to thank the King Abdulaziz City for Science and Technology (KACST) for their 331 generous funding through the Targeted Research Program (TRP) (grant numbers 09-1 and 5-20-

332 01-002-0008). We also would like to thank King Abdulaziz University (KAU) and King Abdullah 333 University of Science and Technology (KAUST) for their continuous support.

334

335 Conflict of interest

336 None 
337 Figure 1. SARS-CoV-2 recombinant proteins. Recombinant SARS-CoV-2 (a) S1 or (b) N

338 proteins were detected by Western blot using anti-His tag antibodies, known seropositive COVID-

33919 human samples or known seronegative COVID-19 human samples. All experiments showed

340 protein bands with expected sizes ( 110 KD and $\sim 46 \mathrm{KD}$ for $\mathrm{S} 1$ and $\mathrm{N}$, respectively).

342 Figure 2. Cut-off values for the developed ELISAs. A 100 serum samples from healthy controls

343 collected before the COVID-19 pandemic were used to determine the cut-off values for (a) S1

344 IgG-ELISA, (b) rS1 IgM-ELISA, (c) N IgG-ELISA and (d) N IgM ELISA. Values were calculated

345 as mean $+3 \mathrm{SD}$. The square is a serologically positive sample from COVID patient. The dotted

346 lines represent the cut-off of each assay.

348 Figure 3. Specificity of the developed ELISAs. Developed ELISAs were tested for their 349 specificity using sera known to be seronegative for SARS-CoV-2 and MERS-CoV $(\mathrm{HC} ; \mathrm{n}=8)$, 350 seropositive sera for MERS-CoV (MERS; $\mathrm{n}=2$ ) or seropositive sera for SARS-CoV-2 (COVID$35119 ; \mathrm{n}=3$ ). These serum samples were also tested for their reactivity in IgG and IgM ELISAs 352 developed for MERS-CoV S1 and N proteins, as well as full S protein from hCoV-OC43, hCoV353 NL63, hCoV-229E, and hCoV-HKU1 viruses. The dotted lines represent the cut-off of each assay.

Figure 4. Humoral immune response to COVID-19. Serum samples from healthy controls $(\mathrm{n}=$

356 125) or COVID-19 patients collected during the first week $(n=10)$, second week $(n=23)$, third 357 week $(n=14)$ or $4^{\text {th }}$ week $(n=5)$ of symptoms onset were tested for IgG and IgM against SARS358 CoV-2 S1 ( $a$ and $b$ ) and N ( $\mathrm{c}$ and $d$ ) proteins using the developed ELISA. The dotted lines represent 359 the cut-off of each assay. 
361 Fig. 5. Receiver operating characteristics (ROC) analysis. ROC analysis was applied to positive 362 vs. negative SARS-CoV-2 samples as identified by RT-PCR assay for (a) S1 IgG-ELISA, (b) S1 363 IgM-ELISA, (c) N IgG-ELISA and (d) N IgM ELISA. Serum samples from healthy controls $(\mathrm{n}=$ 364 125) or COVID-19 patients collected during the first week $(n=10)$, second week $(n=23)$, third 365 week $(n=14)$ or $4^{\text {th }}$ week $(n=5)$ of symptoms onset as well as all COVID-19 samples $(n=52)$. 
404

405

406

407

408

409

410

\section{References}

[1]. Zhu N, Zhang D, Wang W, Li X, Yang B, Song J, et al. A novel coronavirus from patients with pneumonia in China, 2019. N Engl J Med 2020. doi:10.1056/NEJMoa2001017.

[2]. Masters PS. The Molecular Biology of Coronaviruses. Adv Virus Res 2006;65:193-292. doi:10.1016/S0065-3527(06)66005-3.

[3]. Masters PS. The Molecular Biology of Coronaviruses. Adv Virus Res 2006. doi:10.1016/S0065-3527(06)66005-3.

[4]. Fehr AR, Perlman S. Coronaviruses: An overview of their replication and pathogenesis. Coronaviruses Methods Protoc., 2015, p. 1-23. doi:10.1007/978-1-4939-2438-7_1.

[5]. Tan YJ, Lim SG, Hong W. Characterization of viral proteins encoded by the SARScoronavirus genome. Antiviral Res 2005. doi:10.1016/j.antiviral.2004.10.001.

[6]. Hashem AM, Al-amri SS, Al-subhi TL, Siddiq LA, Hassan AM, Alawi MM, et al. Development and validation of different indirect ELISAs for MERS-CoV serological testing. J Immunol Methods 2019. doi:10.1016/j.jim.2019.01.005.

[7]. Degnah AA, Al-amri SS, Hassan AM, Almasoud AS, Mousa M, Almahboub SA, et al. Seroprevalence of MERS-CoV in healthy adults in western Saudi Arabia, 2011-2016. J Infect Public Health 2020. doi:10.1016/j.jiph.2020.01.001.

[8]. Lou B, Li T, Zheng S, Su Y, Li Z, Liu W, et al. Serology characteristics of SARS-CoV-2 infection since the exposure and post symptoms onset. MedRxiv 2020. doi:10.1101/2020.03.23.20041707.

[9]. Yan R, Zhang Y, Li Y, Xia L, Guo Y, Zhou Q. Structural basis for the recognition of SARS-CoV-2 by full-length human ACE2. Science (80- ) 2020. doi:10.1126/science.abb2762.

[10]. Trivedi S, Miao C, Al-Abdallat MM, Haddadin A, Alqasrawi S, Iblan I, et al. Inclusion of MERS-spike protein ELISA in algorithm to determine serologic evidence of MERS-CoV infection. J Med Virol 2018. doi:10.1002/jmv.24948.

[11]. Amanat F, Nguyen T, Chromikova V, Strohmeier S, Stadlbauer D, Javier A, et al. A serological assay to detect SARS-CoV-2 seroconversion in humans. MedRxiv 2020. doi:10.1101/2020.03.17.20037713.

[12]. Xu Y, Xiao M, Liu X, Xu S, Du T, Xu J, et al. Significance of Serology Testing to Assist Timely Diagnosis of SARS-CoV-2 infections: Implication from a Family Cluster. Emerg Microbes Infect 2020. doi:10.1080/22221751.2020.1752610.

[13]. Okba NMA, Muller MA, Li W, Wang C, GeurtsvanKessel CH, Corman VM, et al. SARSCoV-2 specific antibody responses in COVID-19 patients. MedRxiv 2020. doi:10.1101/2020.03.18.20038059.

[14]. Guo L, Ren L, Yang S, Xiao M, Chang D, Yang F, et al. Profiling Early Humoral Response to Diagnose Novel Coronavirus Disease (COVID-19). Clin Infect Dis 2020. doi:10.1093/cid/ciaa310.

[15]. Alshukairi AN, Khalid I, Ahmed WA, Dada AM, Bayumi DT, Malic LS, et al. Antibody response and disease severity in healthcare worker MERS survivors. Emerg Infect Dis 2016. doi:10.3201/eid2206.160010.

[16]. Meyer B, Drosten C, Müller MA. Serological assays for emerging coronaviruses: Challenges and pitfalls. Virus Res 2014. doi:10.1016/j.virusres.2014.03.018.

[17]. Bao L, Deng W, Gao H, Xiao C, Liu J, Xue J, et al. Reinfection could not occur in SARSCoV-2 infected rhesus macaques. BioRxiv 2020. doi:10.1101/2020.03.13.990226. 
411 [18]. Braun J, Loyal L, Frentsch M, Wendisch D, Georg P, Kurth F, et al. Presence of SARSCoV-2 reactive T cells in COVID-19 patients and healthy donors. MedRxiv 2020. doi:10.1101/2020.04.17.20061440.

414

[19]. Wilder-Smith A, Teleman MD, Heng BH, Earnest A, Ling AE, Leo YS. Asymptomatic SARS coronavirus infection among healthcare workers, Singapore. Emerg Infect Dis 2005. doi:10.3201/eid1107.041165.

[20]. Pan X, Chen D, Xia Y, Wu X, Li T, Ou X, et al. Asymptomatic cases in a family cluster with SARS-CoV-2 infection. Lancet Infect Dis 2020. doi:10.1016/S1473-3099(20)301146.

[21]. Wang Y, Liu Y, Liu L, Wang X, Luo N, Ling L. Clinical outcome of 55 asymptomatic cases at the time of hospital admission infected with SARS-Coronavirus-2 in Shenzhen, China. J Infect Dis 2020. doi:10.1093/infdis/jiaa119.

[22]. Yong SEF, Anderson DE, Wei WE, Pang J, Chia WN, Tan CW, et al. Connecting clusters of COVID-19: an epidemiological and serological investigation. Lancet Infect Dis 2020. doi:10.1016/s1473-3099(20)30273-5.

[23]. Du L, He Y, Zhou Y, Liu S, Zheng BJ, Jiang S. The spike protein of SARS-CoV - A target for vaccine and therapeutic development. Nat Rev Microbiol 2009. doi:10.1038/nrmicro2090.

[24]. Vijaykrishna D, Smith GJD, Zhang JX, Peiris JSM, Chen H, Guan Y. Evolutionary Insights into the Ecology of Coronaviruses. J Virol 2007. doi:10.1128/jvi.02605-06.

[25]. Li Y, Yao L, Li J, Chen L, Song Y, Cai Z, et al. Stability issues of RT-PCR testing of SARS-CoV-2 for hospitalized patients clinically diagnosed with COVID-19. J Med Virol 2020. doi:10.1002/jmv.25786.

[26]. An J, Liao X, Xiao T, Qian S, Yuan J, Ye H, et al. Clinical characteristics of the recovered COVID-19 patients with re-detectable positive RNA test. MedRxiv 2020. doi:10.1101/2020.03.26.20044222. 
437 Table 1. Specificity and sensitivity of the developed ELISAs based on sample time collection.

\begin{tabular}{llllll}
\hline \multirow{2}{*}{ ELISA } & Specificity & \multicolumn{2}{l}{ Sensitivity (\%) } & \\
\cline { 3 - 6 } & $(\%)$ & Week 1 & Week 2 & Week 3 & Week 4 \\
\hline S1 IgG & 97.6 & 40 & 88.5 & 100 & 100 \\
S1 IgM & 97.6 & 20 & 84.6 & 100 & 100 \\
N IgG & 91.2 & 60 & 100 & 100 & 100 \\
N IgM & 94.4 & 30 & 88.5 & 78.6 & 60 \\
\hline
\end{tabular}

438 
439 Table 2. Area under the ROC curve (AUC) for the different developed ELISAs based on 440 sample time collection.

441

\begin{tabular}{|c|c|c|c|c|}
\hline$\overline{\text { ELISA }}$ & Samples & $\mathbf{A U C} \pm \mathbf{S D}$ & $95 \% \mathrm{CI}$ & $P$ value \\
\hline \multirow{5}{*}{ S1 IgG } & All samples & $0.940 \pm 0.024$ & $0.892-0.986$ & $<0.0001$ \\
\hline & Week 1 samples & $0.746 \pm 0.091$ & $0.567-0.925$ & 0.0099 \\
\hline & Week 2 samples & $0.973 \pm 0.020$ & $0.935-1.000$ & $<0.0001$ \\
\hline & Week 3 samples & $1.000 \pm 0.000$ & $1.000-1.000$ & $<0.0001$ \\
\hline & Week 4 samples & $1.000 \pm 0.000$ & $1.000-1.000$ & 0.0002 \\
\hline \multirow{5}{*}{ S1 IgM } & All samples & $0.963 \pm 0.014$ & $0.935-0.990$ & $<0.0001$ \\
\hline & Week 1 samples & $0.829 \pm 0.052$ & $0.727-0.931$ & 0.0006 \\
\hline & Week 2 samples & $0.990 \pm 0.007$ & $0.977-1.000$ & $<0.0001$ \\
\hline & Week 3 samples & $1.000 \pm 0.000$ & $1.000-1.000$ & $<0.0001$ \\
\hline & Week 4 samples & $1.000 \pm 0.000$ & $1.000-1.000$ & 0.0002 \\
\hline \multirow{5}{*}{ N IgG } & All samples & $0.971 \pm 0.015$ & $0.942-1.000$ & $<0.0001$ \\
\hline & Week 1 samples & $0.863 \pm 0.065$ & $0.736-0.990$ & 0.0001 \\
\hline & Week 2 samples & $0.994 \pm 0.005$ & $0.985-1.000$ & $<0.0001$ \\
\hline & Week 3 samples & $1.000 \pm 0.000$ & $1.000-1.000$ & $<0.0001$ \\
\hline & Week 4 samples & $1.000 \pm 0.000$ & $1.000-1.000$ & 0.0002 \\
\hline \multirow{5}{*}{ N IgM } & All samples & $0.871 \pm 0.035$ & $0.803-0.940$ & $<0.0001$ \\
\hline & Week 1 samples & $0.528 \pm 0.111$ & $0.311-0.746$ & 0.7655 \\
\hline & Week 2 samples & $0.982 \pm 0.009$ & $0.965-1.000$ & $<0.0001$ \\
\hline & Week 3 samples & $0.929 \pm 0.038$ & $0.854-1.000$ & $<0.0001$ \\
\hline & Week 4 samples & $0.884 \pm 0.067$ & $0.753-1.000$ & 0.0037 \\
\hline
\end{tabular}




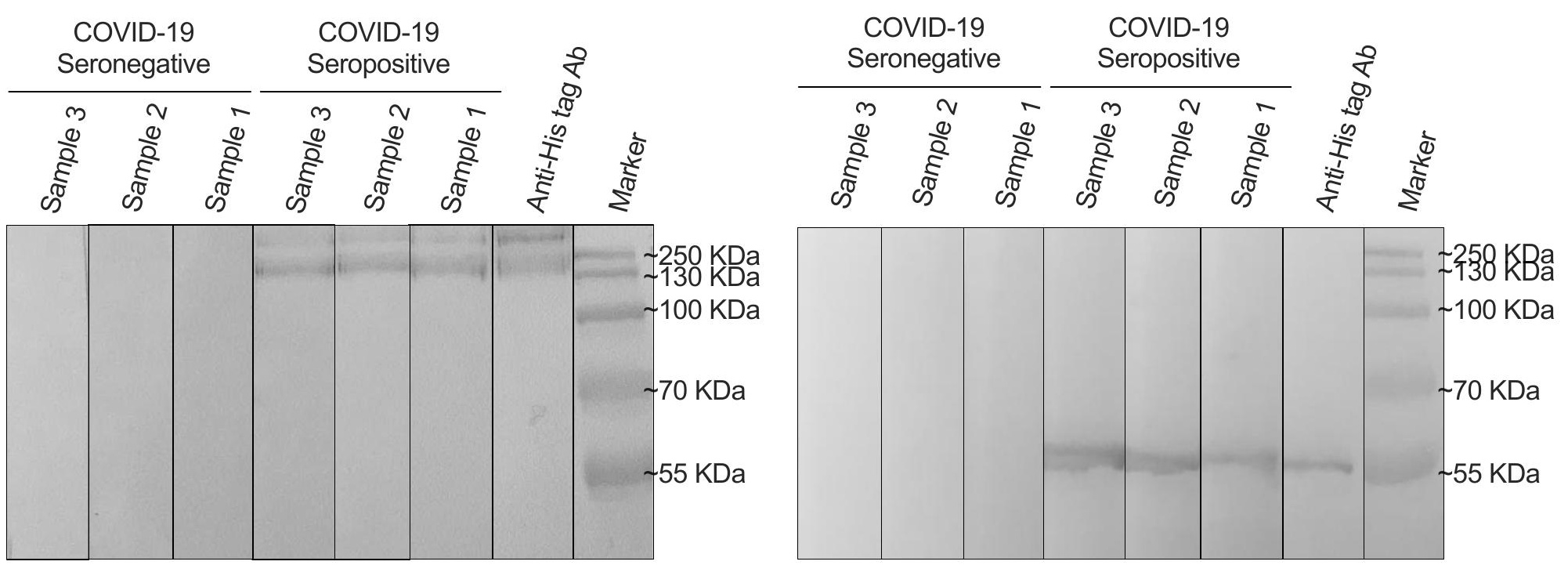



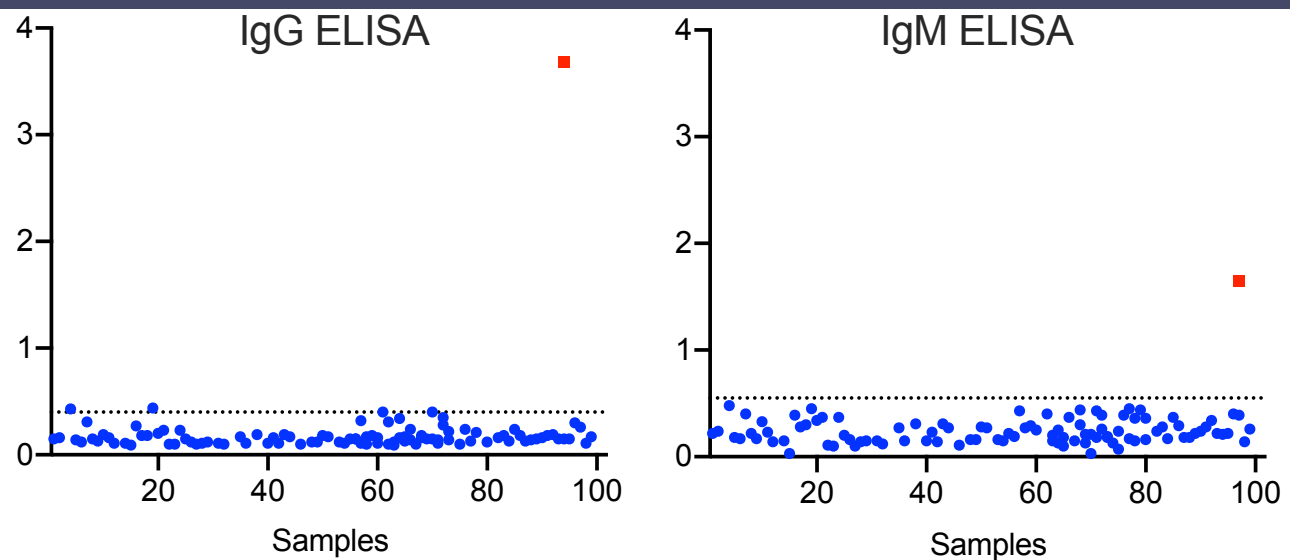

Samples

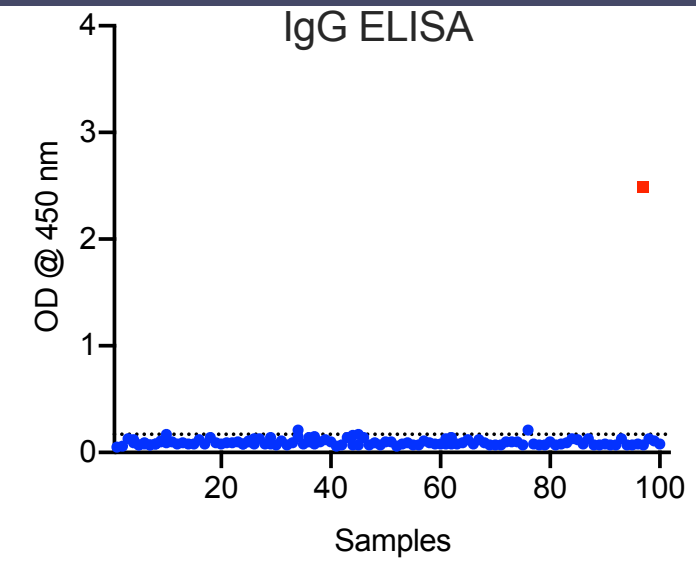

Samples

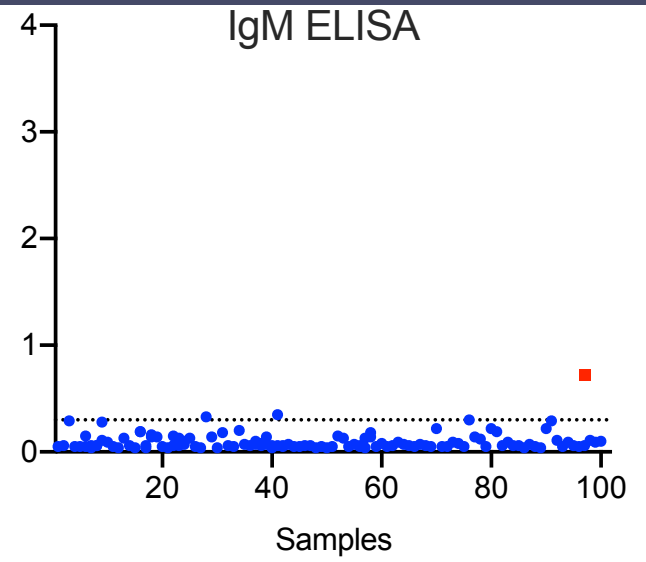

Samples 


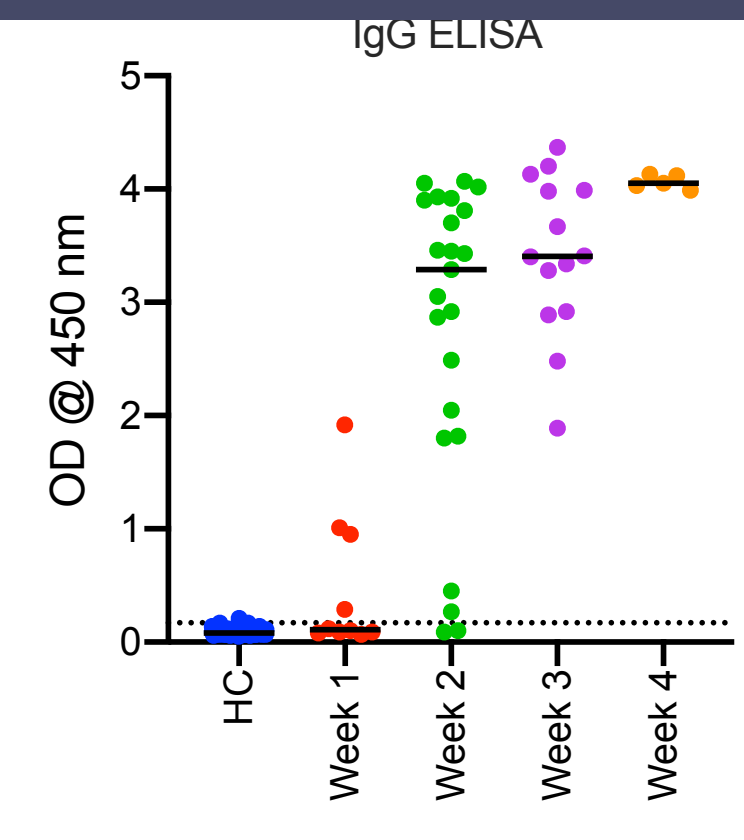

COVID-19 patients

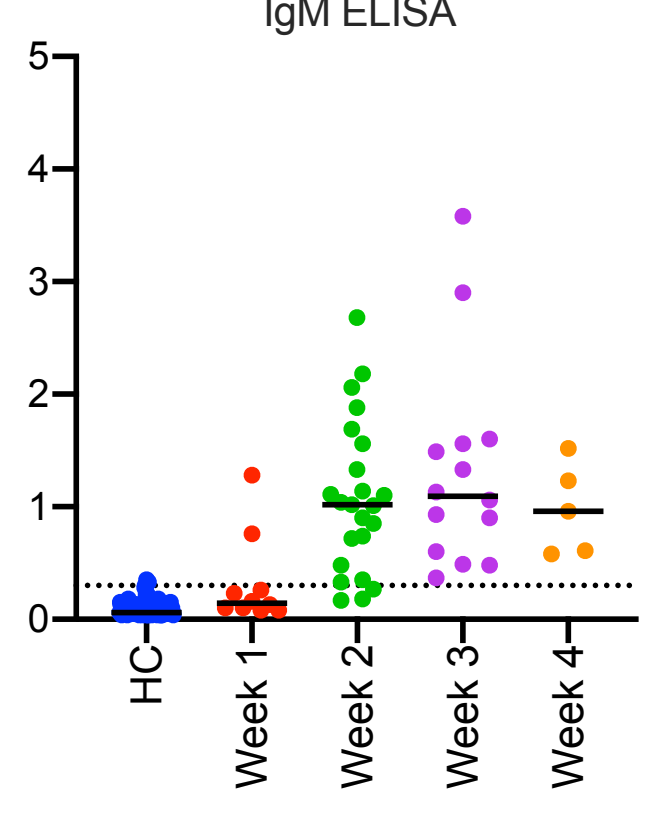

$\overline{\text { COVID-19 patients }}$

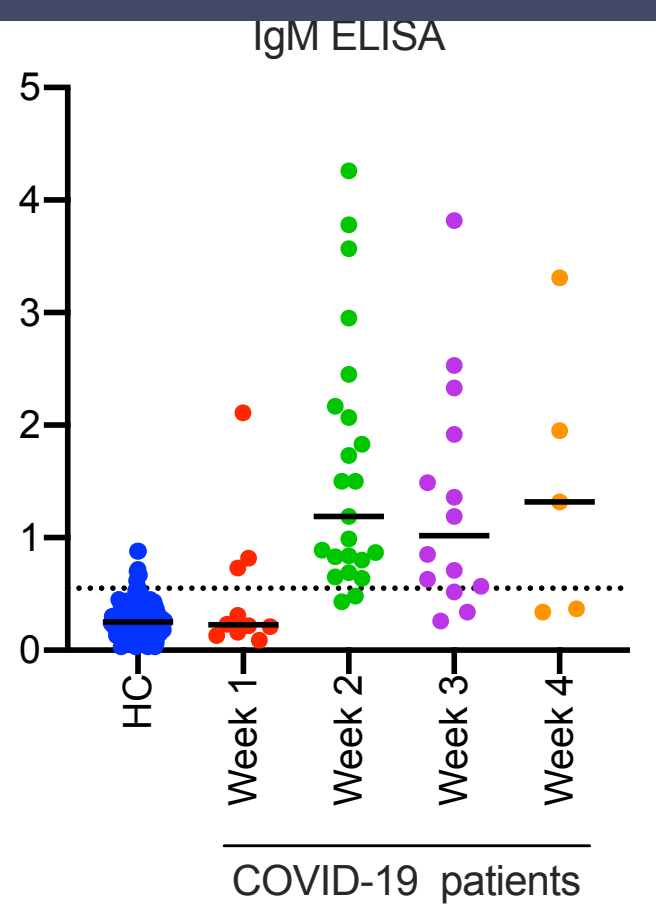

COVID-19 patients
COVID-19 patients

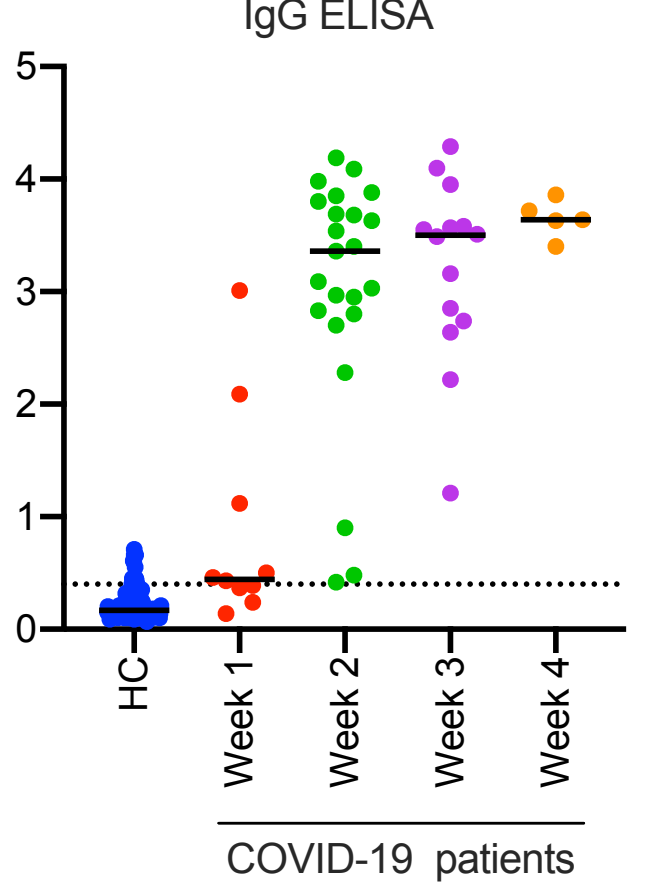

\section{IgM ELISA}



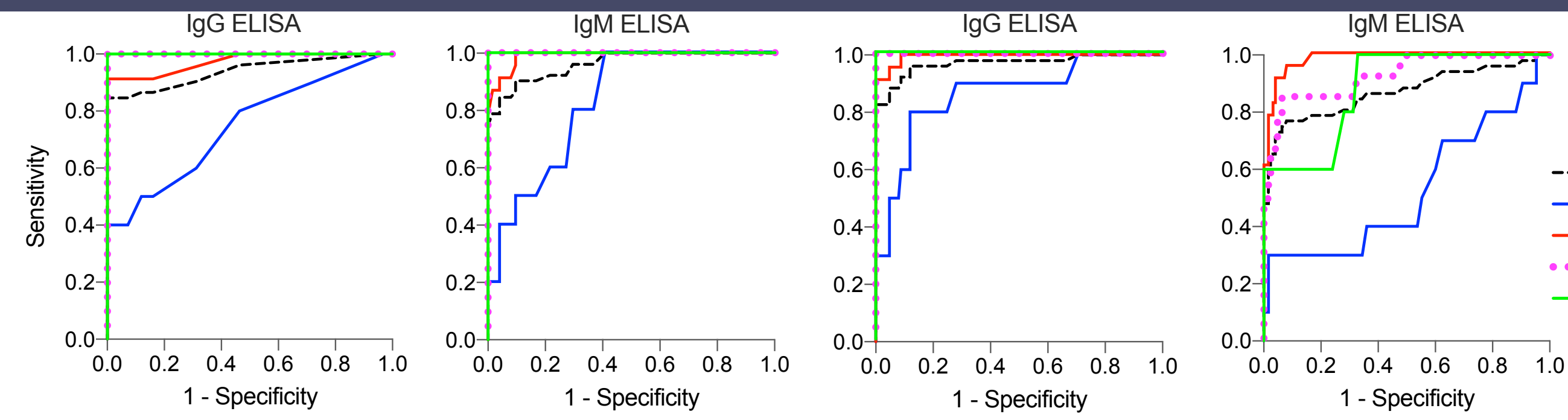

-- All samples

- Week 1 samples

- Week 2 samples

-. Week 3 samples

- Week 4 samples 1 - Specificity 